\title{
GNSS-based Orbital Filter for Earth Moon Transfer Orbits
}

\author{
Vincenzo Capuano, Francesco Basile, Cyril Botteron and \\ Prof. Pierre- André Farine \\ (École Polytechnique Fédérale de Lausanne (EPFL), Switzerland) \\ (E-mail: vincenzo.capuano@epfl.ch)
}

\begin{abstract}
Numerous applications, not only Earth-based, but also space-based, have strengthened the interest of the international scientific community in using Global Navigation Satellite Systems (GNSSs) as navigation systems for space missions that require good accuracy and low operating costs. Indeed, already successfully used in Low Earth Orbits (LEOs), GNSSbased navigation systems can maximise the autonomy of a spacecraft while reducing the burden and the costs of ground operations. That is why GNSS is also attractive for applications in higher Earth orbits up to the Moon, such as in Moon Transfer Orbits (MTOs). However, the higher the altitude the receiver is above the GNSS constellations, the poorer and the weaker are the relative geometry and the received signal powers, respectively, leading to a significant navigation accuracy reduction. In order to improve the achievable GNSS performance in MTOs, we consider in this paper an adaptive orbital filter that fuses the GNSS observations with an orbital forces model. Simulation results show a navigation accuracy significantly higher than that attainable individually by a standalone GNSS receiver or by means of a pure orbital propagation.
\end{abstract}
KEYWORDS
1. GNSS-based Orbital Filter.
2. GNSS.
3. Lunar Missions.
4. Kalman Filter.

Submitted: 2 July 2015. Accepted: 28 October 2015. First published online: 26 November 2015.

1. INTRODUCTION. Although originally designed to provide position, velocity and timing for land, maritime and air applications, Global Navigation Satellite Systems (GNSS) have also been adopted for use in Low Earth Orbit (LEO) space applications such as for attitude determination, time synchronisation, orbit determination, and absolute and relative position determination. Indeed, a GNSS receiver can maximise the autonomy of a spacecraft and reduce the burden and the costs of network operations (Miller, 2011). For the same reasons, the use of GNSS is also very appealing for applications at higher altitudes, such as for Medium Earth Orbit (MEO), Geostationary Orbit (GEO), and Highly Elliptical Orbit (HEO) missions, and even for Moon Transfer Orbits (MTOs), which are the focus of this paper. In particular, the recent stronger interest in lunar exploration in the scientific community has 
pushed companies and institutions to investigate the use of GNSS as the navigation system for Moon missions, as it would significantly reduce the operating costs.

The first space-borne GNSS receiver flew on board the Landsat 4 spacecraft in 1982 (Bauer et al., 2006), while the first use of GNSS above the Global Positioning System (GPS) constellation altitude up to the GEO altitude was made in 1997 during different experiments on board the Equator-S and the DSCS-3 (Defense Satellite Communications 3) satellites, as published in Powell et al. (1999) and Balbach et al. (1998). In more recent papers (Capuano et al., 2013; 2014; 2015; Silva et al., 2013; Palmerini et al., 2009), the use of GNSS as a navigation system to reach the Moon has been analysed. In Capuano et al. (2014) we have shown some preliminary results from the development undertaken in our laboratory of a proof of concept prototype Field-Programmable Gate Array (FPGA)-based GPS receiver for lunar missions.

A GNSS unfiltered navigation solution is approximately as accurate in LEO as on the Earth; above the GNSS constellation on the way to the Moon, however, the accuracy of the system will decrease. Since the GNSS transmitters point towards the Earth, the signal received by a spacecraft above the GNSS constellation will only come from the spill over around the Earth of the signals transmitted from the main lobe or from one of the side lobes of the satellite's antennae patterns. In any case, the received power at the receiver position will be considerably lower than as received by a user on the Earth's surface. This is partly due to the propagation range between the receiver and the GPS satellites that is much higher (in most of the orbit) than for an Earth-based user resulting in extra free space loss attenuation. Moreover if signals are tracked from one of the side lobes of the GNSS antenna pattern the transmitted power is much lower than that transmitted by the main lobe (Unwin et al., 2013). As a result, the number of visible satellites that can be tracked above the GNSS constellation drops dramatically and in some cases it may not be possible to compute a navigation solution (i.e., when there are fewer than four visible satellites). In addition, the poor relative geometry of the GNSS satellites at very high altitude can drastically reduce the navigation solution accuracy, due to the very limited region in the field of view where the GNSS satellites can be observed. This results in an increased Geometric Dilution Of Precision (GDOP) as compared to an Earth user.

On the other hand, a spacecraft is constrained to move along a certain trajectory by the orbital forces acting on it. For these reasons the use of orbital filters, which fuse GNSS observations and the prediction of the space dynamics leads to better solutions than that achieved by a stand-alone GPS receiver. Orbital filters are widely used to improve the accuracy of the navigation solution provided by GNSS receivers. This is documented in Chiaradia et al. (2000), Habib (2013), Pardal et al. (2009) and many others. All of them use a Kalman Filter estimator for the positioning of a satellite in LEO.

In this research, we describe the implementation of GNSS-based orbital filter for lunar missions that uses an adaptive Extended Kalman Filter (EKF) tuned along the MTO as function of the GNSS measurements. Firstly, we consider the use of the GPS L1 Coarse/Acquisition (C/A) signals by modelling the observables as they would be provided by the space-borne WeakHEO receiver prototype (Capuano et al., 2014), an FPGA-based receiver for lunar missions under development in our laboratory. In addition to considering the case of GPS L1 C/A only, we also show the performance achievable by using a dual constellation receiver that processes signals from both GPS and from Galileo. The achieved navigation performance provided by filtering the GNSS observations is compared to the performance obtained by 
using the corresponding non-filtered GNSS least square solution, whose accuracy decreases as the distance from the GNSS constellation increases, becoming almost meaningless when the spacecraft reaches the Moon altitude. Furthermore, we also report the Doppler shift and Doppler rate estimation accuracy of the filter that can strongly aid the GNSS signal processing module.

2. REFERENCE RECEIVER TRAJECTORY. As mentioned in the introduction, the reference trajectory of the receiver is a MTO. The initial conditions of the MTO in the Earth Centred Inertial (ECI) reference frame, the starting date, and the characteristics of the spacecraft assumed in the performed simulations are shown in Table 1 . The motion of the receiver is propagated by the Spirent simulation software SimGen, which models several perturbing accelerations, such as the Earth gravitational spherical harmonics up to the 21st degree and 21st order, the effect of atmospheric drag and Solar Radiation Pressure (SRP), and the third body perturbation due to both the Sun and the Moon (Spirent, 2012).

Figure 1 illustrates the spacecraft trajectory for the entire lunar mission, together with the GPS constellation, that starts from the launch and ends with a selenocentric orbit. The considered trajectory includes only the MTO, which is identified by the light blue orbit portion.

Figure 2 displays the relation between the altitude (of the spacecraft and of the GPS satellites) and the time of the full considered MTO. We can see that for most of the transfer orbit the spacecraft is above the GPS constellation.

3. GNSS OBSERVATIONS MODEL. In order to reproduce correctly the GNSS signal powers at the receiver, the SimGen software of our Spirent 8000 simulator models the gain pattern of both the transmitting and receiving antennas. This is required to differentiate the power level of the signals coming from the side lobes of the transmitting antennae from the ones transmitted from the main lobe.

Information about the transmitting antenna pattern of different GPS signals and blocks are reported in the literature: e.g. for the Block IIA in Czopek and Shollenberger (1993), IIR in Wu (2002) and IIF in Erker et al. (2009). Unfortunately, less detail can be found for Galileo; indeed only the gain at boresight and at the end of the coverage of the transmitting antenna of the four Galileo InOrbit Validation (IOV) satellites are provided in Arenas et al. (2011). Given the missing detailed information about the transmitting antenna patterns for both constellations, we choose for this study that focuses on the orbital filtering performances (and not specifically on the differences between antenna pattern of different blocks and constellations) to adopt the GPS transmitter antenna pattern from Block II-A (illustrated in Figure 3 as defined in Czopek and Shollenberger (1993) and provided by Spirent (2012) to model all the transmitters for all the considered satellite signals. This simplifying assumption, reasonable for the goal of this research, should be considered as an analysis limitation for the reader who is specifically interested in the difference between the performance of the signals transmitted by the side lobes of different GPS and Galileo blocks. In addition, we assumed that the L1 C/A signals are transmitted by 30 GPS satellites and the E5 (E5aQ + E5bQ) signals are transmitted by all the nominal 27 Galileo satellites. 
Table 1. Initial conditions for the MTO, spacecraft characteristics and final altitude before starting the injection into selenocentric orbit manoeuvre.

\begin{tabular}{llll}
\hline Parameter & \multicolumn{3}{c}{ Values } \\
\hline ECI Initial position $(\mathbf{k m})$ & {$\left[\begin{array}{llll}2395 \cdot 52 & -5298 \cdot 28 & -3022 \cdot 82\end{array}\right]$} \\
ECI Initial velocity $(\mathbf{k m} / \mathbf{s})$ & {$\left[\begin{array}{llll}10 \cdot 19 & 3 \cdot 58 & 1 \cdot 72\end{array}\right]$} \\
Initial altitude $(\mathbf{k m})$ & 175 & & \\
Final altitude $(\mathbf{k m})$ & 382912 & \\
Departure date & 2 2nd Jul 2005 & $00: 34: 18$ \\
Mass of the spacecraft $(\mathbf{k g})$ & 1000 & \\
Reference surface $\left(\mathbf{m}^{\mathbf{2}}\right)$ & 20 & \\
\hline
\end{tabular}

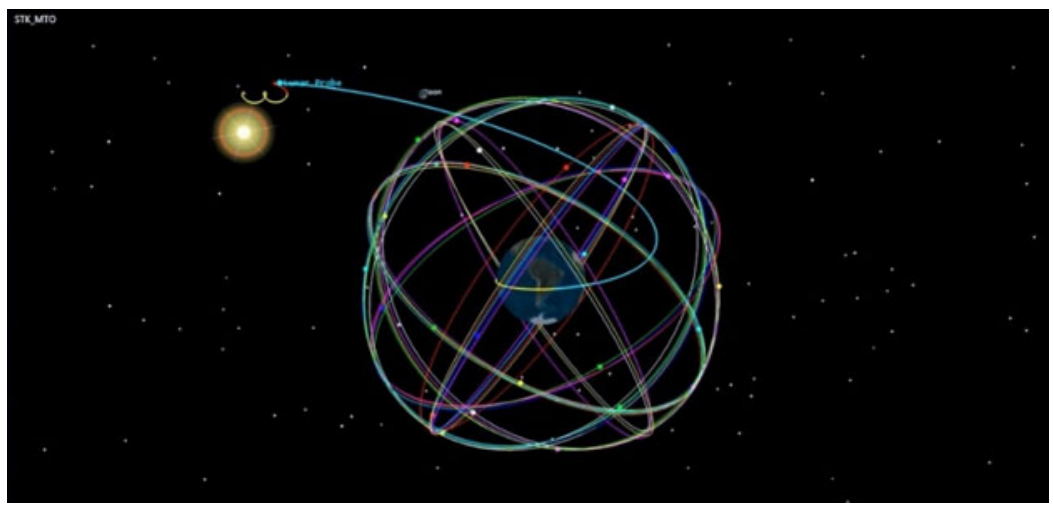

Figure 1. Considered lunar mission: the MTO is the curve in light blue (image generated by using STK).

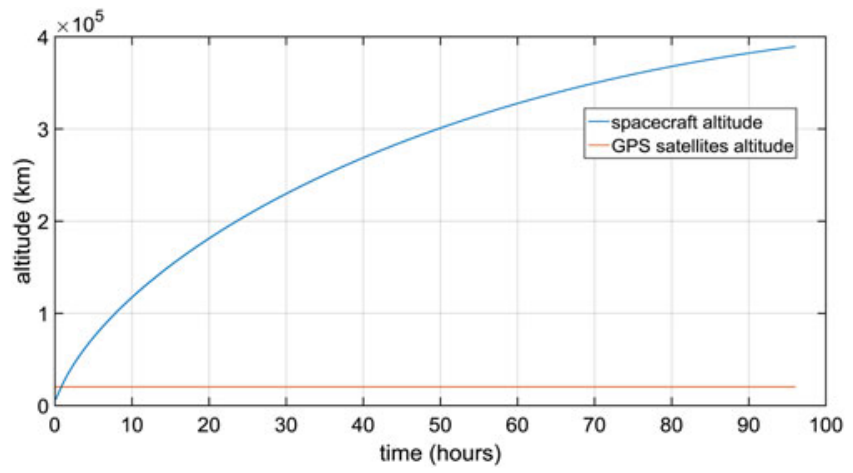

Figure 2. Relation between altitude and time during the considered MTO. The average distance between an Earth receiver and the GPS satellites at zenith is approximately 20,200 km, but for $99 \%$ of the travel time of a receiver flying in the defined MTO, this distance is larger.

Regarding the receiving antenna, following Capuano et al. (2014; 2015), an antenna gain at the receiver of $10 \mathrm{dBi}$ is assumed. Such gain value during the whole trajectory may be obtained placing more than one receiver antenna on different faces of the 


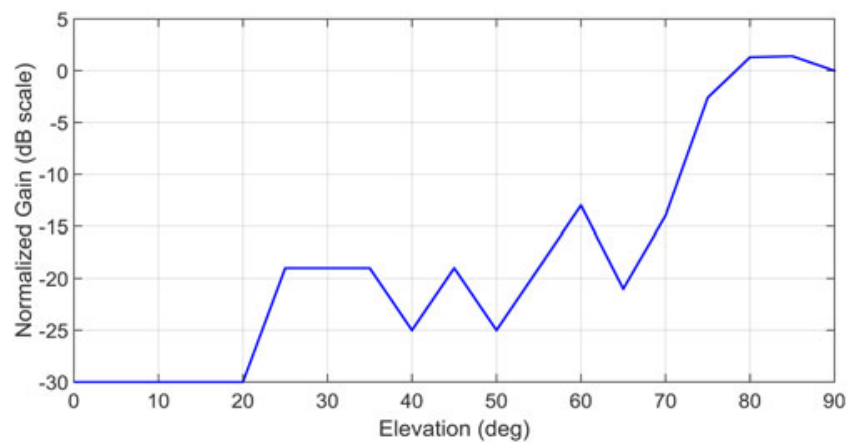

Figure 3. GPS Transmitter Antenna Pattern used to simulate the antenna pattern of all considered GNSS satellites (based on Czopek and Shollenberger (1993) for Block II-A). The boresight is at $90^{\circ}$. The gain is normalised to $0 \mathrm{~dB}$ at boresight.

spacecraft (in such a way that at least one points to the GNSS satellites), as assumed in Capuano et al. (2014; 2015) and Palmerini et al. (2009), or by using a steerable directive antenna (Litva, 1996).

In order to compute for each satellite a realistic signal power $P_{r, k}$ at the receiver, where $k$ denotes the satellite number, SimGen simulates for each satellite the received GNSS signal strength using the following equation (Spirent, 2012):

$$
P_{r, k}=P_{I C D}+O_{G}+20 \times \log _{10}\left(\frac{R_{0}}{R_{k}}\right)-L_{t x}-L_{r x}
$$

where $P_{I C D}$ is the guaranteed minimum signal level on the Earth as given in GNSS Signal In Space Interface Control Documents (SIS ICDs). $O_{G}$ is the "Global Offset". A value of $+3 \mathrm{~dB}$ has been used to match the performance obtained when using the simulator (transmitting $P_{I C D}$ ) with the performance obtained when real signals are received. Since the transmitted signal powers are typically from 1 to $5 \mathrm{~dB}$ higher than the minimum received signal power value (Kaplan and Hegarty, 2006), an intermediate value of $3 \mathrm{~dB}$ has been chosen for both constellations. $R_{0}$ is the reference range used for inverse-square variation calculation and equal to the range from an Earth-based receiver to the GNSS satellite at zero elevation. $R_{k}$ is the range from the $k$ th GNSS satellite to the receiver. $L_{t x, k}$ is the gain from the $k$ th GNSS satellite transmit antenna in the direction of the receiver that takes into account the radiation pattern of the antenna. Finally, $L_{r x, k}$ is the gain from the receiver antenna in the direction of the $k$ th GNSS satellite which in our paper has been considered as constant and equal to $10 \mathrm{dBi}$.

Since the simulator only supports 12 channels for the GPS constellation and 12 channels for the Galileo constellation, a selection of the simulated satellites are performed by SimGen. For a high orbit mission (where the signals are very weak), the 12 simulated satellites from each constellation are those corresponding to the 12 strongest received signals (Spirent, 2012).

As stated in our previous studies (Capuano et al., 2014; 2015), for the considered MTO, the minimum receiver sensitivity required to acquire and track at least the four most powerful signals from the GPS satellites simultaneously is about -168 $\mathrm{dBm}$ (at least four satellites are required to compute the navigation solution). 
Therefore, by considering a receiver antenna gain of $10 \mathrm{dBi}$, we are considering a receiver sensitivity of $-159 \mathrm{dBm}$, taking a $1 \mathrm{~dB}$ margin. The received power value in $\mathrm{dBm} P_{r}$ can also be expressed in terms of carrier-to-noise ratio $C / N_{0}$, which is given by the following equation, considering a front-end noise figure of $2 \mathrm{~dB}$ and assuming an effective antenna temperature of $130 \mathrm{~K}$ (Van Diggelen, 2009).

$$
C / N_{0}=P_{r}+174
$$

Using Equation (2) the sensitivity value of $-159 \mathrm{dBm}$ corresponds to $15 \mathrm{~dB}-\mathrm{Hz}$.

3.1. GPS observations. For the GPS L1 C/A signals, we have used $P_{I C D}=$ $-128 \cdot 5 \mathrm{dBm}$ according to (Anon., 2011).

In our simulations, the pseudorange and pseudorange rate observables from the visible signals are modelled according to the GPS theory presented in Kaplan and Hegarty (2006) as follows:

$$
\begin{gathered}
\rho=\sqrt{\left(x_{\text {sat }}-x_{u}\right)^{2}+\left(y_{\text {sat }}-y_{u}\right)^{2}+\left(z_{\text {sat }}-z_{u}\right)^{2}}+b+\operatorname{errors}_{\rho} \\
\dot{\rho}=\left(\boldsymbol{v}_{\text {sat }}-\boldsymbol{v}_{\boldsymbol{u}}\right) \cdot \boldsymbol{a}+\dot{b}+\text { errors }_{\dot{\rho}}
\end{gathered}
$$

In Equation (3), $\left[\begin{array}{lll}x_{\text {sat }} & y_{\text {sat }} & z_{\text {sat }}\end{array}\right]^{T}$ denotes the position's vector of the GPS satellite that is transmitting the signal, $\left[\begin{array}{lll}x_{u} & y_{u} & z_{u}\end{array}\right]^{T}$ is the user's position vector, and $b$ is the receiver's clock offset in metres. We assumed an arbitrary initial clock offset of $10 \mathrm{~km}$. In Equation (4), $\boldsymbol{v}_{\boldsymbol{s} \boldsymbol{a}}$ and $\boldsymbol{v}_{\boldsymbol{u}}$ are, respectively, the velocity vector of the transmitting GPS satellite and of the spacecraft, $\dot{b}$ represents the clock's drift expressed as rangerate bias (in $\mathrm{m} / \mathrm{s}$ ), and $\boldsymbol{a}$ is the Line-Of-Sight (LOS) vector from the user to the GPS satellite. A clock drift of $\dot{b}=100 \mathrm{~m} / \mathrm{s}$ has been considered (note that this is a conservative value as a more precise clock such as an Oven Controlled Oscillator (OCXO) can achieve a frequency variation of about one part in $10^{11}$ over a second, corresponding to a range-rate bias on the order of $3 \mathrm{~m} / \mathrm{s}$ (Groves, 2013). Both the position and velocity of the GPS satellites and of the receiver are provided by Spirent's simulator.

Pseudorange observables are affected by systematic and non-systematic errors denoted as errors $\rho_{\rho}$ in Equation (3), which can be classified into: Signal-in-Space Ranging Error (SISRE), which includes satellite clock error and broadcast satellite ephemeris error; atmospheric delay; multipath effect and receiver error.

According to Kaplan and Hegarty (2006), these errors can be assumed as white Gaussian noise with a certain standard deviation (although this is not strictly true it is sufficient for this analysis), as summarised in Table 2 and described below. The overall error that affects pseudoranges can thus be described by the user equivalent range error $\left(\sigma_{U E R E}\right)$, defined as the root sum square of the different range error contributions in Table 2.

According to McDonald and Hegarty (2000), for the GPS constellation we have considered a value of $0.5 \mathrm{~m}$ for the transmitter's clock and broadcast ephemeris errors often described as Signal-in-Space Ranging Error (SISRE) (Engel, 2008).

The residual error on pseudorange measurements due to ionospheric effect is considered only when the spacecraft is inside the atmosphere. When the receiver is located above $1000 \mathrm{~km}$, which is the edge of the ionosphere (Kaplan and Hegarty, 2006), the GPS signals may cross the ionosphere only when they are transmitted by satellites which are on the other side of the Earth. In this case the ionosphere layer could be crossed twice with a consequently greater delay of the signals. However when the 
Table 2. GPS L1 C/A code error budget. $h$ denotes the altitude of the spacecraft, and $\sigma_{\mathrm{tDLL}}$ denotes the DLL (Delay Lock Loop) code thermal noise jitter that depends on the received $\mathrm{C} / \mathrm{No}$

\begin{tabular}{ll}
\hline Error source & $1 \sigma$ error $(\mathrm{m})$ \\
\hline Signal-in-Space Ranging Error (SISRE) & $0 \cdot 5$ \\
Ionospheric delay & 7 if $h<1000 \mathrm{~km}$ \\
Receiver error and resolution & $\left(0 \cdot 1^{2}+\sigma_{t D L L}^{2}\right)^{1 / 2}$ \\
Multipath & $0 \cdot 2$ \\
\hline
\end{tabular}

receiver is far enough from the Earth (i.e. in most parts of the MTO up to $384400 \mathrm{~km}$ ), it rarely receives signals that, transmitted from GNSS satellites at MEO altitudes of roughly 19,000-23,000 km altitude, cross the $\sim 19-23$ times smaller ionosphere layer. Therefore, in this study, the ionosphere delay residual of $7 \mathrm{~m}$ (Kaplan and Hegarty, 2006) is only modelled when the receiver is below the altitude of the ionosphere, while when above, pseudoranges from satellites whose line of sight crosses the ionosphere (and thus the troposphere situated below) are discarded. Furthermore, in this simulation, when the receiver is below the highest bound of the ionosphere the signals never pass through the troposphere, so the troposphere is neglected.

Typical modern Earth GNSS receivers have values for the pseudorange noise and resolution error of approximately $0 \cdot 1 \mathrm{~m}$ or less $(1 \sigma)$ in nominal conditions (Kaplan and Hegarty, 2006). However, for very weak signals such as those seen when operating above the GPS constellation, the noise value can be much higher. The DLL (Delay Lock Loop) code thermal noise jitter $\sigma_{\mathrm{tDLL}}$ increases as the receiver carrier to noise density of the signal $C / N_{0}$ decreases (Kaplan and Hegarty, 2006). For this reason we have modelled the $\sigma_{\mathrm{tDLL}}$ as a function of the $C / N_{0}$ and we have added it to the constant value of $0 \cdot 1 \mathrm{~m}$ that conservatively takes into account other possible error sources. For Binary Phase Shift Keying (BPSK) modulations (e.g., GPS L1 C/A and Galileo E5 individually filtered components), when a non-coherent power DLL discriminator is used, a general expression for the thermal noise code tracking jitter is according to Betz and Kolodziejski (2000):

$\sigma_{\mathrm{tDLL}} \cong$

$$
\left\{\begin{array}{l}
\sqrt{\frac{B_{n}}{2 \frac{C}{N_{0}}} D\left[1+\frac{2}{\left.T \frac{C}{N_{0}}(2-D)\right]}\right]}, D \geq \frac{\pi R_{c}}{B_{f e}} \\
\sqrt{\frac{B_{n}}{2 \frac{C}{N_{0}}\left(\frac{1}{B_{f e} T_{c}}\right)\left[1+\frac{1}{T \frac{C}{N_{0}}}\right]}, D \leq \frac{R_{c}}{B_{f e}} \quad[\text { chips }]} \\
\sqrt{\frac{B_{n}}{2 \frac{C}{N_{0}}}\left[\left(\frac{1}{B_{f e} T_{c}}\right)+\frac{B_{f e} T_{c}}{\pi-1}\left(D-\frac{1}{B_{f e} T_{c}}\right)^{2}\right]\left[1+\frac{C}{\left.T \frac{C}{N_{0}}(2-D)\right]}\right]}, \quad \frac{R_{c}}{B_{f e}}<D<\frac{\pi R_{c}}{B_{f e}}
\end{array}\right.
$$


where $B_{n}$ is the code loop noise bandwidth expressed in $\mathrm{Hz}$; $D$ is the early-to-late correlator spacing in units of chips; $T$ is the coherent integration time in seconds; $B_{f e}$ is the double-sided front-end bandwidth in $\mathrm{Hz} ; R_{c}=1 / T_{c}$ is the chipping rate expressed in chips/s and $C / N_{0}$ is the carrier to noise ratio in $\mathrm{dB}-\mathrm{Hz}$. In our simulations, we have assumed a $B_{n}=0.5 \mathrm{~Hz}, D=0.3$ chips, $T=20 \mathrm{~ms}, B_{f e}=26 \mathrm{MHz}, R_{c}=1 / T_{c}=1.023$ Mchip/s (values chosen in order to match the current parameters setting of the space-borne GPS-based WeakHEO receiver prototype under development in our laboratory (Capuano et al., 2014)).

Modern GNSS receivers obtain pseudorange rate observables by evaluating the Doppler shift of the received frequency from the transmitted one. As stated in Kaplan and Hegarty (2006), pseudorange rates may be computed simply by multiplying the Doppler shift with the wavelength of the signal carrier. This is done here; hence, the pseudorange rate error errors $\dot{\rho}$ in Equation (4) is due to the error in the frequency estimation. In particular, Doppler frequency estimations (and then pseudorange rates) are also affected by thermal noise, which is assumed here as the only source of error. According to (Borio et al., 2010) and assuming a standard PLL (Phase Lock Loop), the standard deviation of the Doppler tracking jitter is

$$
\sigma_{f}=\frac{1}{T} \sqrt{\frac{B_{n}}{C / N_{0}}\left(1+\frac{1}{2 T C / N_{0}}\right)}\left[\frac{r a d}{s}\right]
$$

Note that the velocity can also be obtained taking successive phase measurements when they are available and differentiating with time, giving a more accurate measure, which is less sensitive to the tracking loop jitter.

3.1.1. Standalone GPS L1 ClA least square positioning solution. Figure 4 highlights the performance of the GPS L1 C/A stand-alone receiver, in terms of 3D position error when a least square estimator is used to compute the position from the pseudorange observations. The 3D position error increases as the spacecraft gets closer to the Moon, reaching peaks of more than $50 \mathrm{~km}$, which clearly does not satisfy the required positioning accuracy of less than $1 \mathrm{~km}(3 \sigma)$ for a Moon mission (Woodward and Folta, 2009).

On one hand, the increasing error trend is due to pseudorange error, which grows because of the increasing code tracking thermal noise as shown in Figure 5 that plots the pseudorange error as provided by one of the 12 channel outputs of the Spirent simulator as a function of the altitude. This is mainly due to the carrier-tonoise ratio $C / N_{0}$, which becomes lower and lower as the distance from the transmitting satellites increases. In fact the pseudorange error, in particular the code tracking thermal jitter range error, strictly depends on the $C / N_{0}$, as shown in Equation (5). On the other hand, the very high peaks in the 3D position error are due to the corresponding peaks of the GDOP as shown by comparing Figure 4 with Figure 6 that provides the GDOP as a function of altitude. In particular, such discontinuities of the GDOP can be explained by the following two considerations. Firstly, because of the limited number of channels supported (12 per GNSS constellation), the simulator selects only the 12 strongest signals, without taking into account if they are transmitted by satellites leading to a bad geometrical distribution; secondly, as explained above, a signal may suddenly be discarded by the positioning algorithm because it starts crossing the ionosphere, with a sudden impact on the GDOP. 


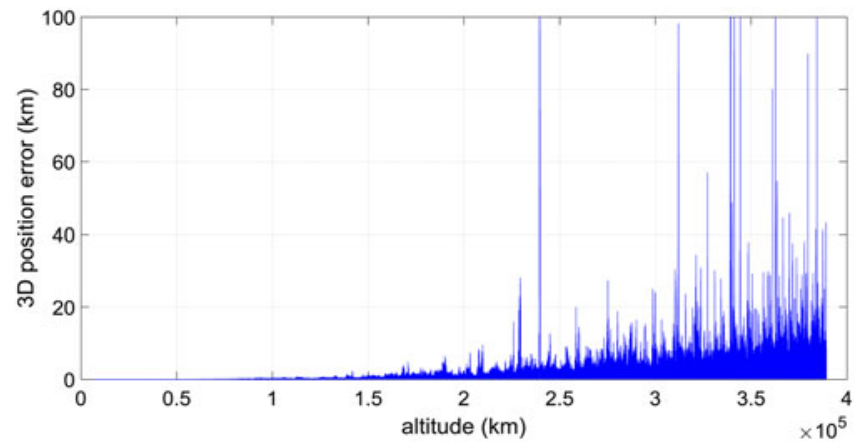

Figure 4. 3D positioning error, for GPS C/A, as function of the altitude.

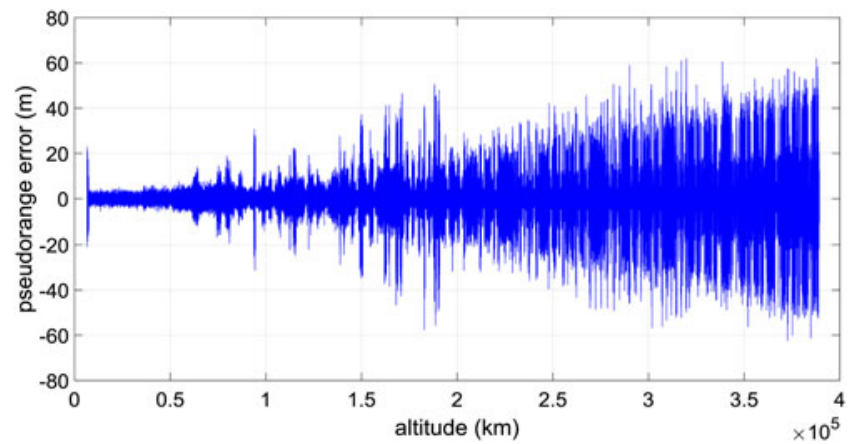

Figure 5. Pseudorange error for one of the 12 channel outputs of the Spirent simulator as a function of the altitude. Note that different satellites are simulated at different times within a given channel.

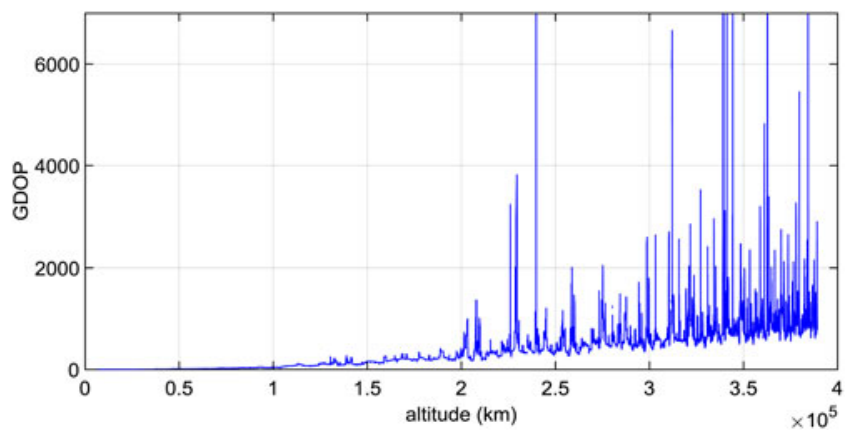

Figure 6. GDOP as function of the altitude.

3.2. GPS-Galileo combined observations. In order to investigate the performance achievable when using a GPS-Galileo combined constellation, we have chosen to process the observables obtained from the wideband Galileo E5aQ + E5bQ pilot signals in addition to the GPS L1 C/A signal, as done in Capuano et al. (2015). 
In particular, we have selected the Galileo E5 pilot signals as their chipping rate is ten times higher than that of the E1 signals, thus leading to a reduced tracking error in the ranging measurements. Additionally, the use of the pilot channels enables very long coherent integration times which is desired in very high sensitivity scenarios (as the coherent integration time for data channels is typically limited to one bit duration to avoid the losses incurred by the bit transitions). Note also that once a pilot channel is successfully tracked, it is easier to acquire and estimate the navigation data bits from the data channel since both channels are fully synchronized.

Similarly as for GPS, the realistic power levels at the receiver position of the Galileo E5a + E5b signals are computed by the SimGen software based on Equation (1) using a guaranteed minimum signal level on the Earth $P_{I C D}=-125 \mathrm{~dB}$ according to ESA (2010).

All the other assumptions for the error budget on pseudorange and pseudorange rate measurements from the Galileo E5a + E5b signals correspond to the same ones presented in Section 3.1 for GPS L1 C/A, except for the error induced by the space segment (the SISRE), which has been set to $0.65 \mathrm{~m}$ according to Engel (2008).

The performances obtainable by using solely the Galileo observations as input to a least square estimator are not reported here since they present similar characteristics to those obtained for GPS only; however, more details about the use of the Galileo only constellation can be found in our previous study (Capuano et al., 2015).

4. ORBITAL FILTER. The proposed orbital filter is based on the well-known Kalman Filter (KF) algorithm (Kalman, 1960). The spacecraft dynamics model is the system model of the filter used to predict the GNSS range and range rate measurements model. Because of the highly nonlinearity of both system and measurement model, the EKF is adopted. The continuous time system model and the measurement model are respectively represented by:

$$
\dot{\boldsymbol{x}}(t)=\boldsymbol{f}(\boldsymbol{x}(t))+\boldsymbol{G}(t) \boldsymbol{w}_{\boldsymbol{s}}(t)
$$

and

$$
\boldsymbol{z}(t)=\boldsymbol{h}(\boldsymbol{x}(t))+\boldsymbol{w}_{\boldsymbol{m}}(t),
$$

where $\boldsymbol{x}(t)$ is the state vector, $\boldsymbol{f}(\boldsymbol{x}(t))$ is a nonlinear function of the state vector, $\boldsymbol{G}(t)$ is the system noise distribution matrix, $\boldsymbol{w}_{\boldsymbol{s}}(t)$ is the system noise vector, $\boldsymbol{z}(t)$ is the measurement vector, $\boldsymbol{h}(\boldsymbol{x}(t))$ is a nonlinear function of the state vector used to predict the observation and $\boldsymbol{w}_{\boldsymbol{m}}(t)$ is the measurement noise vector.

Table 3 shows the Kalman filter algorithm (Groves, 2013), where $\hat{\boldsymbol{x}}_{k}^{-}$is the a priori state estimate at a time step $k, \hat{\boldsymbol{x}}_{k-1}^{+}$is the a posteriori state estimate at a time step $k-1$, $\boldsymbol{\Phi}_{k-1}$ is the state transition matrix at a time step $k-1, \boldsymbol{P}_{k}^{-}$is the a priori estimate error covariance at a time step $k, \boldsymbol{P}_{k-1}^{+}$is the a posteriori estimate error covariance at a time step $k-1, \boldsymbol{Q}_{k-1}$ is the discrete process noise covariance a time step $k-1, \boldsymbol{R}_{k}$ is the discrete measurement noise covariance at a time step $k, \boldsymbol{H}_{k}$ is the measurement matrix at a time step $k, \boldsymbol{K}_{k}$ is the Kalman gain at a time step $k, \boldsymbol{z}_{k}$ is the measurement vector at a time step $k, \delta z_{k}^{-}$is the innovation measurement vector at a time step $k$ and $\boldsymbol{I}$ is a unit matrix. 
Table 3. Extended Kalman Filter (EKF) algorithm for navigation.

\begin{tabular}{ll}
\hline Quantity & \multicolumn{1}{c}{ Formulation } \\
\hline Predicted state vector & $\hat{\boldsymbol{x}}_{k}^{-}=\hat{\boldsymbol{x}}_{k-1}^{+}+\int_{k-1}^{k} \boldsymbol{f}(\boldsymbol{x}, t) d t$ \\
Predicted system noise covariance matrix & $\boldsymbol{P}_{k}^{-}=\boldsymbol{\Phi}_{k-1} \boldsymbol{P}_{k-1}^{+} \boldsymbol{\Phi}_{k-1}^{T}+\boldsymbol{Q}_{k-1}$ \\
Kalman Gain matrix & $\boldsymbol{K}_{k}=\boldsymbol{P}_{k}^{-} \boldsymbol{H}_{k}^{T}\left(\boldsymbol{H}_{k} \boldsymbol{P}_{k}^{-} \boldsymbol{H}_{k}^{T}+\boldsymbol{R}_{k}\right)^{-1}$ \\
Corrected state estimate & $\hat{\boldsymbol{x}}_{k}^{+}=\hat{\boldsymbol{x}}_{k}^{-}+\boldsymbol{K}_{k}\left(\boldsymbol{z}_{k}-\boldsymbol{h}\left(\hat{\boldsymbol{x}}_{k}^{-}\right)\right)=\hat{\boldsymbol{x}}_{k}^{-}+\boldsymbol{K}_{k} \delta \boldsymbol{z}_{k}^{-}$ \\
Corrected system noise covariance matrix (Joseph form) & $\boldsymbol{P}_{k}^{+}=\left(\boldsymbol{I}-\boldsymbol{K}_{\boldsymbol{k}} \boldsymbol{H}_{k}\right) \boldsymbol{P}_{k}^{-}\left(\boldsymbol{I}-\boldsymbol{K}_{k} \boldsymbol{H}_{k}\right)^{T}+\boldsymbol{K}_{k} \boldsymbol{R}_{k} \boldsymbol{K}_{k}^{T}$ \\
\hline
\end{tabular}

4.1. State vector and measurements vector. The state vector is composed of eight elements; it includes the position and velocity components of the spacecraft, and the receiver's clock offset $b$ and clock drift $\dot{b}$.

$$
\boldsymbol{x}=\left[\begin{array}{llllllll}
x & y & z & b & u & v & w & \dot{b}
\end{array}\right]^{T}
$$

The measurement vector is

$$
\boldsymbol{z}=\left[\begin{array}{c}
\boldsymbol{\rho}_{G P S} \\
\dot{\boldsymbol{\rho}}_{G P S}
\end{array}\right]
$$

where $\boldsymbol{\rho}_{G P S}$ are the pseudoranges of the available GPS satellites and $\dot{\boldsymbol{\rho}}_{G P S}$ are the pseudorange rates of the available GPS satellites.

4.2. Spacecraft dynamics model. Different accelerations are included in the model by three different configurations:

1. below $9600 \mathrm{~km}$ from the centre of the Earth, spherical harmonics of Earth gravitational potential up to $6^{\text {th }}$ degree and $6^{\text {th }}$ order;

2. in between $9600 \mathrm{~km}$ and $50000 \mathrm{~km}$, spherical harmonics up to $2^{\text {nd }}$ degree and $2^{\text {nd }}$ order, the Solar Radiation Pressure (SRP) and the gravitational perturbations due to the Sun and the Moon;

3. above $50000 \mathrm{~km}, 1^{\text {st }}$ order Earth gravity, SRP and lunar and solar third body perturbations.

Although at low altitude the atmospheric drag has a significant effect on a satellite's orbit, it is not modelled since the benefit in the navigation solution's accuracy obtainable by including the drag in the process is not considered worth the computational cost required and in addition, the GNSS stand-alone performance is very accurate in LEO.

The computation of the Earth gravitational potential and the acceleration of the spacecraft due to a second and third perturbing mass (which in our case are the Sun and the Moon) have been modelled according to Montenbruk and Gill (2000) while the effect of the SRP according to Battin (1999). However, more details can be found in Basile (2014).

Figure 7 shows the 3D position error over time if the trajectory is estimated by only integrating the dynamics equations, thus as pure orbital propagation. A typical drift affects the propagation reaching almost $300 \mathrm{~km}$ of error at the end of the MTO. It is important to note that both the GNSS and the orbital propagator systems if used individually provide a very coarse accuracy at the end of the MTO (see 


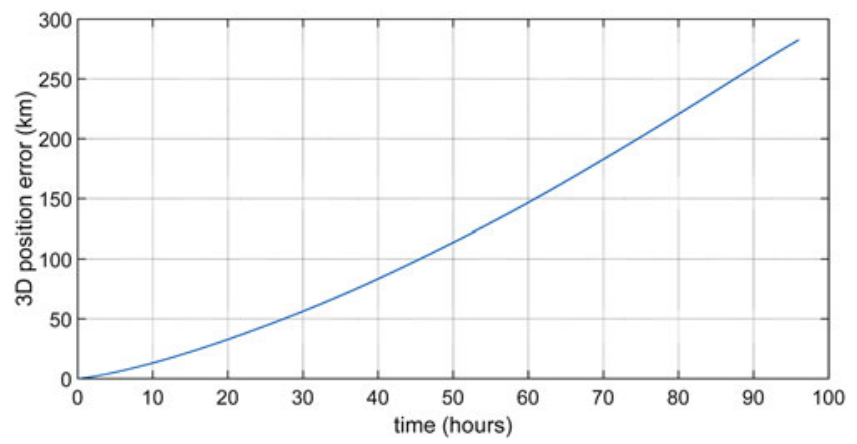

Figure 7. Orbital propagator 3D position error over time for the full MTO.

Figures 4 and 7). However, since the position estimation of both systems is characterised by a different error distribution, their fusion can result in a significant improvement of the individual accuracy (as will be shown in the results of Section 5); in fact the GNSS measurements prevent the orbital propagation solution drifting, while the orbital propagation smooths the GNSS solution and bridges signal outages.

4.3. Observation functions. The GNSS receiver provides $n$ measurements of pseudorange $\rho$ and pseudo-range rate $\dot{\rho}$ from $\mathrm{n}$ different transmitting satellites. These measurements are predicted by the following observation functions of the state vector (Kaplan and Hegarty, 2006):

$$
\begin{gathered}
\rho^{-}=\sqrt{\left(x_{s a t}-x^{-}\right)^{2}+\left(y_{s a t}-y^{-}\right)^{2}+\left(z_{s a t}-z^{-}\right)^{2}}+b^{-} \\
\dot{\rho}^{-}=\left(\boldsymbol{v}_{\text {sat }}-\boldsymbol{v}^{-}\right) \cdot \boldsymbol{a}^{-}+\dot{b}^{-}
\end{gathered}
$$

In Equation (11), $\left[\begin{array}{lll}x_{\text {sat }} & y_{\text {sat }} & z_{\text {sat }}\end{array}\right]^{T}$ denotes the position's vector of the GNSS satellite that is transmitting the signal, $\left[\begin{array}{lll}x^{-} & y^{-} & z^{-}\end{array}\right]^{T}$ is the predicted user's position vector, and $b^{-}$is the predicted receiver's clock offset. In Equation (12), $\boldsymbol{v}_{\boldsymbol{s a t}}$ and $\boldsymbol{v}^{-}$are, respectively, the velocity vector of the transmitting GNSS satellite and the velocity vector of the spacecraft, $\dot{b}^{-}$represents the predicted clock's drift, and $\boldsymbol{a}^{-}$is the predicted line-ofsight (LOS) unit vector from the user to the GNSS satellite.

The predicted observation vector $z^{-}$consists of $2 n$ elements:

$$
\boldsymbol{z}^{-}=\boldsymbol{h}\left(\boldsymbol{x}^{-}\right)=\left[\begin{array}{llllllll}
\rho_{1}^{-} & \rho_{2}^{-} & \cdots & \rho_{n}^{-} & \dot{\rho}_{1}^{-} & \dot{\rho}_{2}^{-} & \cdots & \dot{\rho}_{n}^{-}
\end{array}\right]^{T}
$$

4.4. State transition matrix computation. The state transition matrix $\boldsymbol{\Phi}$ is required to compute the predicted system noise covariance matrix. The transition matrix can be expressed as:

$$
\boldsymbol{\Phi}_{k-1}=\exp \left(\boldsymbol{F}_{k-1} \tau_{s}\right) \cong\left(\boldsymbol{I}+\boldsymbol{F}_{k-1} \tau_{s}\right)
$$

where $\tau_{s}=t_{k}-t_{k-1}$ is the propagation interval, while:

$$
\boldsymbol{F}_{k-1}=\left.\frac{\partial \boldsymbol{f}(\boldsymbol{x})}{\partial \boldsymbol{x}}\right|_{\boldsymbol{x}=\hat{\boldsymbol{x}}_{k-1}^{+}}
$$

As shown in Equation (14), $\boldsymbol{\Phi}$ is a function of the system matrix $\boldsymbol{F}$, which is linearized 
about the state vector estimate (see Equation (15)). To compute the system matrix $\boldsymbol{F}$ linearized about the state vector estimate $\hat{\boldsymbol{x}}^{+}$the complex-step derivative approximation is adopted. This method has been investigated in many works (e.g. Anderson et al., 2001; Lai and Crassidis 2006; Martins et al., 2003).

4.5. Observation matrix. The observation matrix $\boldsymbol{H}$ at a time step $k$ is defined as the Jacobian of the observations defined in Equations (11) and (12):

$$
\boldsymbol{H}_{k}=\left.\frac{\partial \boldsymbol{h}(\boldsymbol{x})}{\partial \boldsymbol{x}}\right|_{\boldsymbol{x}=\hat{\boldsymbol{x}}_{\bar{k}}^{-}}=\left.\frac{\partial \boldsymbol{z}(\boldsymbol{x})}{\partial \boldsymbol{x}}\right|_{\boldsymbol{x}=\hat{\boldsymbol{x}}_{\bar{k}}^{-}} .
$$

Thus, it corresponds to the following $2 n \times 8$ matrix :

$$
\boldsymbol{H}_{k}=\left[\begin{array}{cccccccc}
a x_{1} & a y_{1} & a z_{1} & 1 & 0 & 0 & 0 & 0 \\
a x_{2} & a y_{2} & a z_{2} & 1 & 0 & 0 & 0 & 0 \\
\vdots & \vdots & \vdots & \vdots & \vdots & \vdots & \vdots & \vdots \\
a x_{n} & a y_{n} & a z_{n} & 1 & 0 & 0 & 0 & 0 \\
0 & 0 & 0 & 0 & a x_{1} & a y_{1} & a z_{1} & 1 \\
0 & 0 & 0 & 0 & a x_{2} & a y_{2} & a z_{2} & 1 \\
\vdots & \vdots & \vdots & \vdots & \vdots & \vdots & \vdots & \vdots \\
0 & 0 & 0 & 0 & a x_{n} & a y_{n} & a z_{n} & 1
\end{array}\right]
$$

where $\left[\begin{array}{lll}a_{x j} & a_{y j} & a_{z j}\end{array}\right]^{T}$ represents the LOS vector between the receiver and the jth satellite at a time step $k$. Note that the dependence of the pseudorange rate on the position is not null but it can be considered negligible; in fact for an Earth user a $1 \mathrm{~m}$ position error has an impact on the psudorange rate of only $\sim 5 \times 10^{-5} \mathrm{~m} \mathrm{~s}^{-1}$ (Groves, 2013) and then even less during most of the MTO.

4.6. Adaptivity of the filter. The accuracy of the observations strongly worsens with the altitude along the MTO, as shown in Section 3.1.1. Therefore the covariance matrix $\boldsymbol{R}_{k}$ of the measurements is tuned adaptively as a function of the GNSS measurements error. The adaptive strategy adopted is illustrated in Figure 8. The code tracking thermal noise $\sigma_{t D L L}$ and the Doppler tracking jitter $\sigma_{f}$ (that affect respectively pseudoranges and pseudorange rates) are computed by means of the $C / N_{0}$, by using Equations (5) and (6), respectively. Once $\boldsymbol{R}_{k}$ is computed, as a function of the current $\sigma_{U E R E}$, the EKF provides the navigation solution by fusing the prediction of the dynamics and the GNSS measurements. As stated previously, the GDOP can be very high, resulting in very large position error peaks. In order to remove such large error peaks, the orbital filter makes a check of the GDOP computed by means of the estimated state and, if it exceeds a threshold N (a value of 1500 has been set after tuning), the estimation will only rely on the orbital propagation. Corresponding to GDOP peaks higher than the threshold, the measurements are considered not reliable enough and the orbital propagator is used to bridge the consequent outage. This is not statistically optimal, but for the very short time intervals of the GDOP peaks, it provides higher accuracy. While $\boldsymbol{R}_{k}$ is a function of $\sigma_{U E R E}, \boldsymbol{Q}_{k}$ is constant. The following results have been obtaining setting $\boldsymbol{R}_{k}$ and $\boldsymbol{Q}_{k}$ as reported in Table 4.

4.7. Frequency aiding for the GNSS receiver. A precise estimation of Doppler shifts and Doppler rates that affect the carrier frequency can provide a precious aiding to the signal processing engine as described in Capuano et al. $(2014 ; 2015)$ 


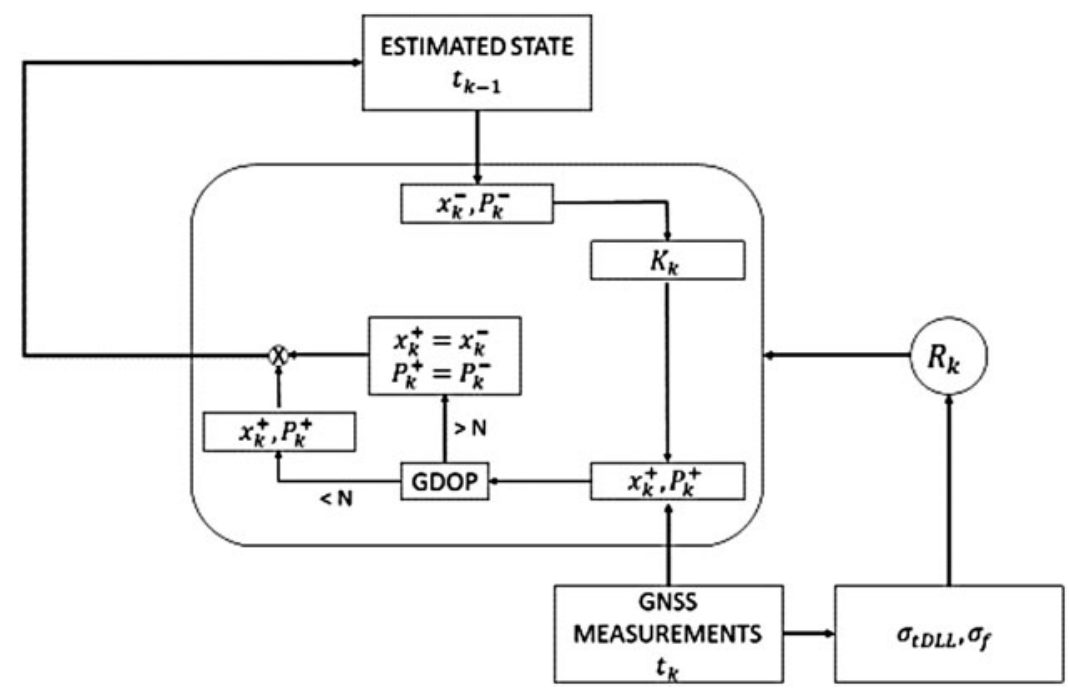

Figure 8. Adaptive strategy.

Table 4. Adopted measurement and process covariance matrices. The symbols $\sigma_{\rho_{i}}$ and $\boldsymbol{\sigma}_{\dot{\rho}_{i}}$ represent respectively the root sum square of the different range error contributions (defined as $\sigma_{\text {UERE }}$ ) and the Doppler tracking jitter defined in Equation (6).

\begin{tabular}{lc}
\hline Covariance Matrices & \multicolumn{1}{c}{ Formulation $\left(\mathrm{m}^{2}\right)$} \\
\hline$R_{k}=$ & $\operatorname{diag}\left({\sigma_{\rho_{1}}}^{2}(t),{\sigma_{\rho_{2}}}^{2}(t), \ldots, \sigma_{\rho_{n}}{ }^{2}(t),{\sigma_{\dot{\rho}_{1}}}^{2}(t),{\sigma_{\dot{\rho}_{2}}}^{2}(t), \ldots,{\sigma_{\dot{\rho}_{n}}}^{2}(t)\right)$ \\
$Q_{k}=$ & $\operatorname{diag}\left(1,1,1,1,10^{-1}, 10^{-1}, 10^{-1}, 10^{-1}\right)$ \\
\hline
\end{tabular}

and Silva et al. (2013). Expected Doppler shifts $\Delta f_{\text {expected }}$ can be computed from the estimated range rate $\dot{r}_{\text {estimated }}$ by means of the more accurate filtered navigation solution and of the GNSS satellites ephemeris as

$$
\Delta f_{\text {expected }}=-\frac{f_{T}}{c} \cdot \dot{r}_{\text {estimated }}
$$

where $f_{T}$ is the transmitted frequency (e.g. 1575.42 MHz for GPS L1) and $c$ is the speed of light.

In addition, expected Doppler rate $\dot{\Delta} f_{\text {expected }}$ can be computed as follows:

$$
\dot{\Delta} f_{\text {expected }}=\frac{\Delta f_{\text {expected }}\left(t_{k+1}\right)-\Delta f_{\text {expected }}\left(t_{k}\right)}{t_{k+1}-t_{k}} .
$$

\section{RESULTS}

5.1. GPS L1 C/A-based orbital filter performance. Figure 9 highlights the accuracy achievable by the implemented GPS L1 C/A-based orbital filter, in terms of 3D position error. The maximum error is about $260 \mathrm{~m}$, more than two orders of magnitude less than the maximum error obtained with the stand-alone GPS receiver. For the last 5 


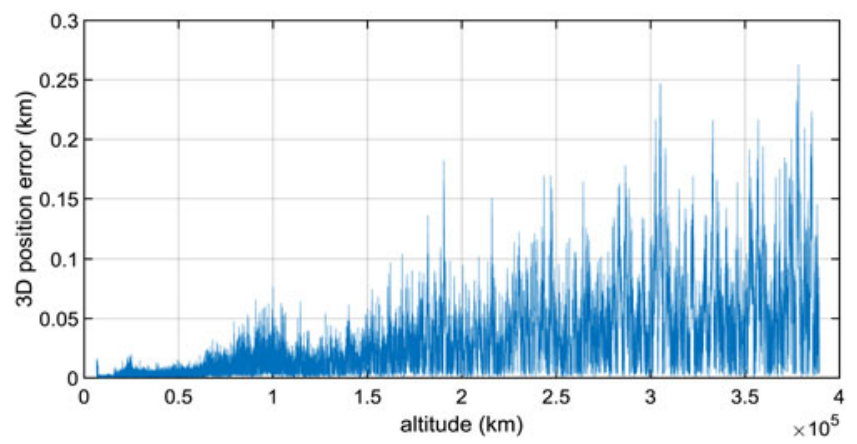

Figure 9. 3D normalised position error obtained with the GPS-based orbital filter.

hours and 45 minutes of orbit (i.e. the last portion where the error does not drift significantly, which starts approximately at $376200 \mathrm{~km}$ altitude), the standard deviation is equal to $80.5 \mathrm{~m}(1 \sigma)$. Similar results have been obtained in (Silva et al., 2013), for a MTO with a minimum $\mathrm{C} / \mathrm{N}_{0}=15 \mathrm{~dB}-\mathrm{Hz}$ and with a minimum $\mathrm{C} / \mathrm{N}_{0}=10 \mathrm{~dB}-\mathrm{Hz}$.

Figure 10 displays the 3D velocity error of the GPS L1 C/A orbital filtered solution. In the last 5 hours and 45 minutes of the MTO, the standard deviation is about 9.51 $\mathrm{cm} / \mathrm{s}(1 \sigma)$.

By considering for example one of the 12 channel outputs of Spirent (the first one) for the last 5 hours and 45 minutes of orbit, the GPS L1 C/A orbital filtered solution can predict the Doppler shift and Doppler rate with an error of about $0.061 \mathrm{~Hz}$ and $0.039 \mathrm{~Hz} / \mathrm{s}$ standard deviation $(1 \sigma)$ respectively, as illustrated for the whole trajectory in Figures 11 and 12.

5.2. Use of GPS-Galileo combined constellation. Figure 13 illustrates the improvement achievable in availability when using a GPS-Galileo combined constellation and Figure 14 the consequent reduction of the GDOP as compared to using GPS only. As a combined result of better availability and smaller GDOP, the performance in the position estimation are improved too, as shown in Figure 15: during the last 5 hours and 45 minutes of the simulation, the standard deviation of the $3 \mathrm{D}$ position error is approximately $9 \mathrm{~m}(1 \sigma)$, one order of magnitude less than that obtained in the single constellation case (see Figure 9).

Figure 16 shows the velocity estimate accuracy, in terms of 3D velocity error, improved too. In the last 5 hours and 45 minutes of trajectory, we obtained about $3.5 \mathrm{~cm} / \mathrm{s}(1 \sigma)$, much better than the GPS only-based orbital filter.

The improvements in the navigation solution lead to a better estimation of the Doppler shift and Doppler rate as well, as shown in Figures 17 and 18, with an error of $0.041 \mathrm{~Hz}$ and $0.021 \mathrm{~Hz} / \mathrm{s}(1 \sigma)$ respectively during the last 5 hours and 45 minutes of considered trajectory.

Adding GLONASS and BeiDou (as future GNSS receivers probably will do) should further improve the GDOP.

6. CONCLUSIONS. Several studies have proved the feasibility of GNSS as a navigation system for high Earth orbits and in particular for Moon missions. Although the very weak GNSS signals at the Moon's altitude can be processed with modern high 


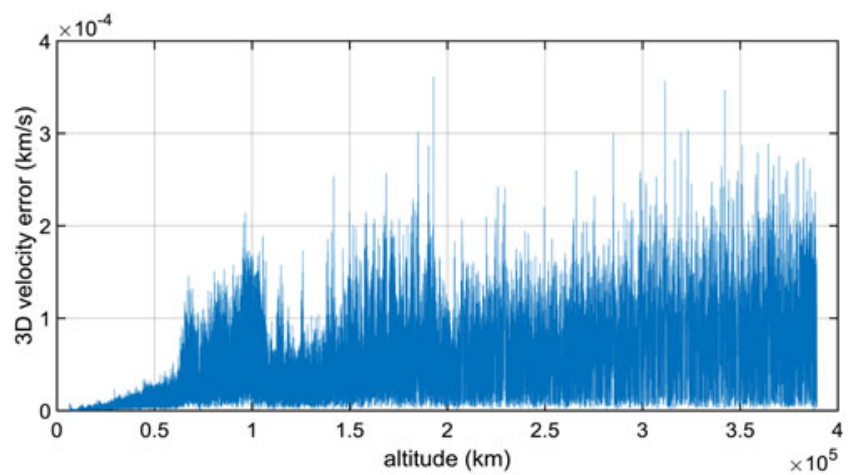

Figure 10. 3D normalised velocity error obtained with the GPS-based orbital filter.

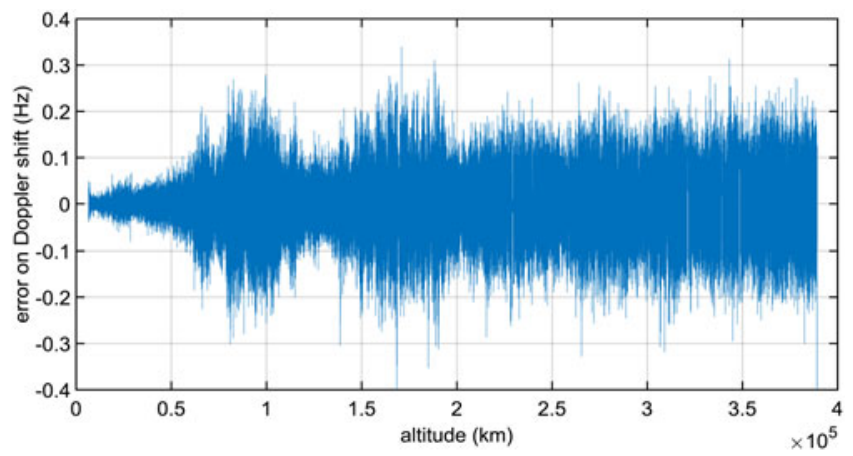

Figure 11. Doppler shift estimation error for the first channel output of Spirent: GPS-based orbital filter.

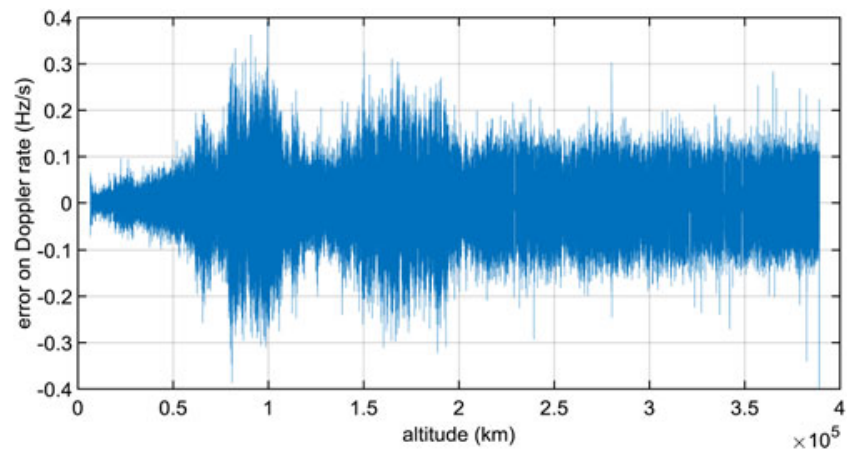

Figure 12. Doppler rate estimation error for the first channel output of Spirent: GPS-based orbital filter. 


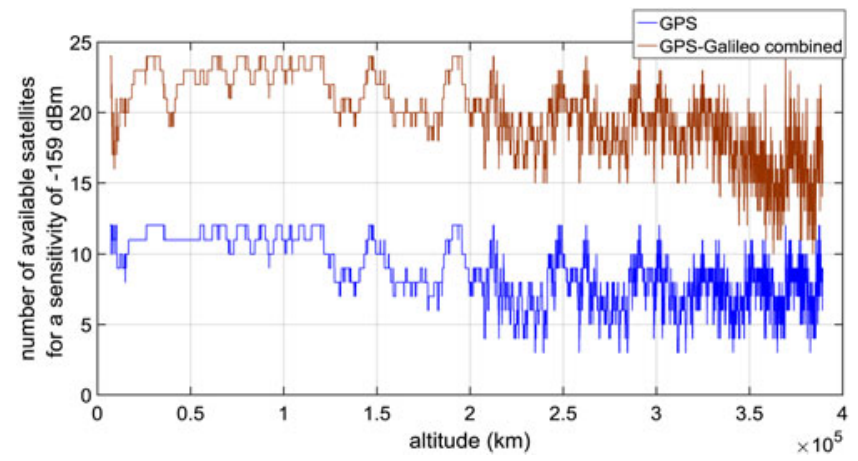

Figure 13. GNSS availability for a single GPS constellation and for a GPS-Galileo combined constellation, for a sensitivity of $-159 \mathrm{dBm}$.

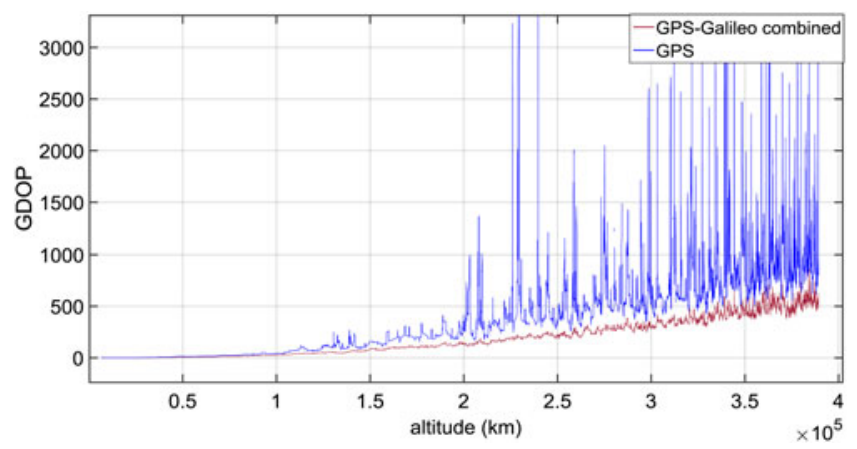

Figure 14. GDOP for a single GPS constellation and for a GPS-Galileo combined constellation, for a sensitivity of $-159 \mathrm{dBm}$.

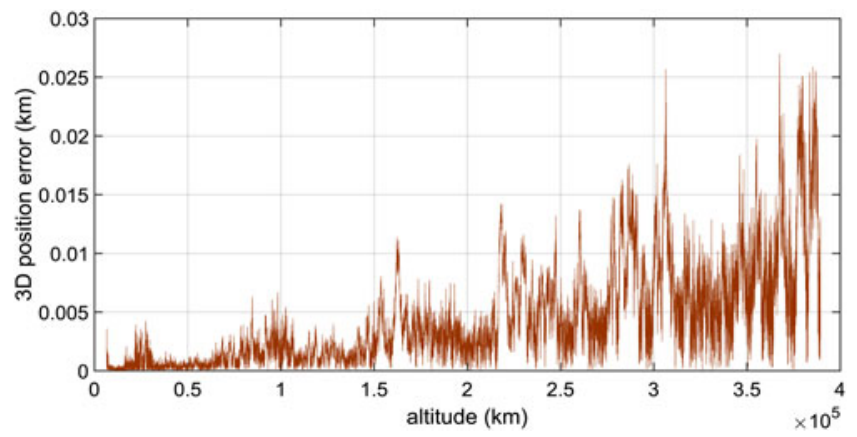

Figure 15. 3D normalised position error obtained with the GPS-Galileo-based orbital filter.

sensitivity GNSS receivers, the accuracy of a standalone GNSS navigation solution would be much lower than that typically required for a Moon mission. In this paper we have investigated the use of an orbital filter, specifically designed for a MTO, which fuses GNSS observations of pseudorange and pseudorange rate with an 


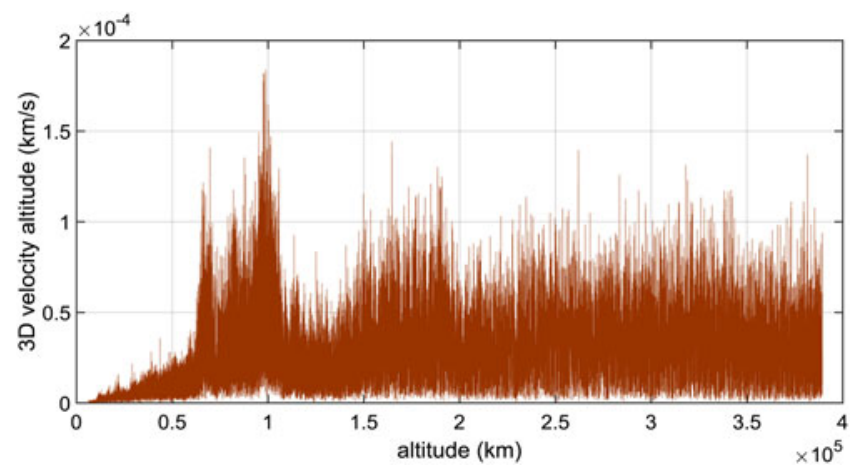

Figure 16. 3D normalised velocity error obtained with the GPS-Galileo-based orbital filter.

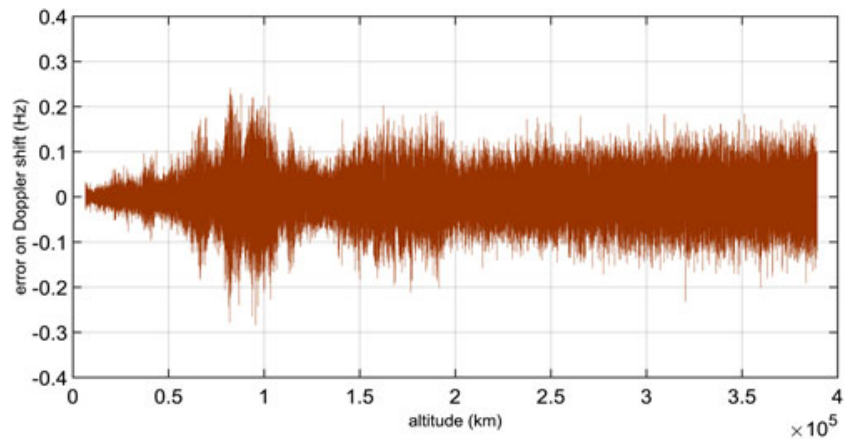

Figure 17. Doppler shift estimation error for the first channel output of Spirent: GPS + Galileobased orbital filter.

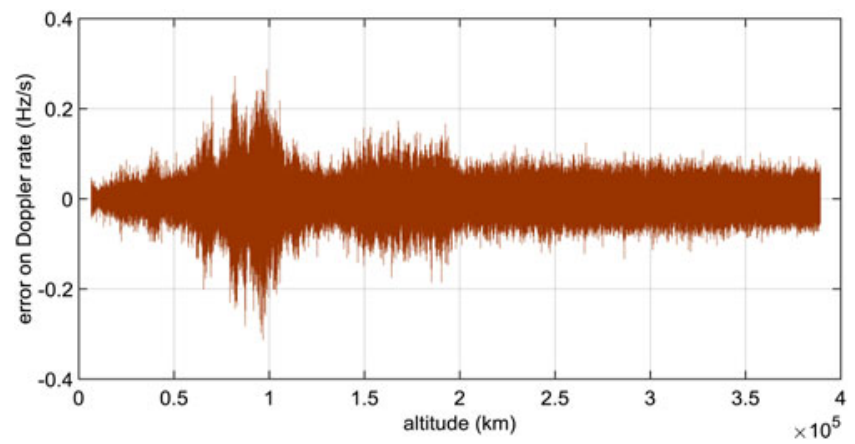

Figure 18. Doppler rate estimation error for the first channel output of Spirent: GPS + Galileobased orbital filter.

orbital forces model through an adaptive EKF. A reference trajectory and a realistic operational scenario have been defined to reproduce a direct MTO of a Moon mission. The positioning error achievable by using a least square estimator of the available GPS observations has been simulated to quantify what would be the accuracy of a 
non-filtered standalone GPS L1 C/A receiver during the whole trajectory. As expected, once the receiver is flying above the GPS constellation, the positioning error increases with the altitude because of a decreasing accuracy of the GNSS measurements. This is due to weaker signals and a worsening of the relative geometry between receiver and transmitters (higher GDOP). Without any kind of filtering tens of km error with peaks higher than $50 \mathrm{~km}$ have been observed in simulation at Moon altitude, assuming a GPS L1 C/A receiver capable of tracking signals down to $-159 \mathrm{dBm}$.

The implementation of our orbital filter has been described, highlighting the importance of its adaptive architecture that takes into account the decreasing accuracy of the GNSS observations when the receiver is orbiting above the GNSS constellation on the route to the Moon. Simulation results have shown a significant improvement of the positioning accuracy when using the orbital filter. The peaks of the error have been reduced to about $260 \mathrm{~m}$ by filtering the same GPS L1 C/A observations used for the least square estimation. Accuracies of tens of $\mathrm{cm} / \mathrm{s}$ have been obtained for the velocity estimation. Position and velocity estimation have also been used to estimate Doppler shift and Doppler rate; very useful to aid the signal processing module of the GNSS receiver. The Doppler shift and Doppler rate estimation errors have a standard deviation of $0.06 \mathrm{~Hz}$ and of $0.04 \mathrm{~Hz} / \mathrm{s}$ at Moon altitude, respectively. Finally we have investigated the improvements achievable when using observations from a GPS-Galileo combined constellation. Significant further improvements are attained in positioning, velocity, Doppler shift and Doppler rate estimation when using the two constellations concurrently. However, without cross-validation using real input data, the results obtained by using the Kalman filter have to be treated cautiously. In order to validate the system experimentally, in future works the orbital filter effectiveness will be tested using measurements provided by our GPS L1 C/A space-borne WeakHEO receiver, under development, specifically designed for lunar missions.

\section{REFERENCES}

Anderson, K.W., Newman, J.C. and Nielsen, E.J. (2001). Sensitivity Analysis for Navier-Stokes Equations on Unstructured Meshes Using Complex Variables. AIAA Journal, 39(1), 56-63.

Anon. (2011). ICD-GPS-200F Navstar GPS Space Segment/User Segment Interfaces.

Arenas, S., Monjas, F., Montesano, A., Montesano, C., Mangenot, C. and Salghetti, L. (2011). Performances of GALILEO system navigation antenna for Global Positioning. Proceedings of the 5th European Conference on Antennas and Propagation (EUCAP).

Balbach, O., Eissfeller, B., Hein, G.W., Enderle, W., Schmidhuber, M. and Lemke, N. (1998). Tracking GPS above GPS satellite altitude: first results of the GPS experiment on the HEO mission Equator-S. Position Location and Navigation Symposium, IEEE., Palm Springs, California, USA. DOI: 10.1109/ PLANS.1998.670065. pp. 243-249.

Basile, F. (2014). Implementation of the orbital filter in a GNSS receiver for lunar missions, Rome: University of Rome La Sapienza.

Battin, R.H. (1999). An Introduction to Mathematics and Methods of Astrodynamics, AIAA Education Series.

Bauer, F.H., Moreau, M.C., Dahle-Melsaether, M.E., Petrofski, W.P., Stanton, B.J., Thomason, S., Harris, G.A, Sena, R.P., and Parker Temple, L., III (2006) The GPS Space Service Volume, Proceedings of the 19th International Technical Meeting of the Satellite Division of The Institute of Navigation (ION GNSS 2006), Fort Worth, TX, pp. 2503-2514.

Betz, J.W. and Kolodziejski, K.R. (2000). Extended Theory of Early-Late Code Tracking for a Bandlimited GPS Receiver. Navigation, 47(3), 211-226.

Borio, D., Sokolova, N. and Lachapelle, G. (2010). Doppler Measurement Accuracy in Standard and HighSensitivity GNSS Receivers. IET Radar, Sonar \& Navigation. 5(6), 657-665. 
Capuano, V., Botteron, C. and Farine, P. A. (2013). GNSS performances for MEO, GEO and HEO. Beijing, China, 64th International Astronautical Congress (IAC).

Capuano, V., Botteron, C., Wang, Y., Tian, J., Leclère, J. and Farine, P.-A. (2014), GNSS/INS/Star Tracker Integrated Navigation System for Earth-Moon Transfer Orbit, Proceedings of the 27th International Technical Meeting of The Satellite Division of the Institute of Navigation (ION GNSS+ 2014), Tampa, Florida, pp. 1433-1447.

Capuano, V., Botteron, C., Leclere, J., Tian, J., Yanguang, W. and Farine, P.-A. (2015). Feasibility study of GNSS as navigation system to reach the Moon. Acta Astronautica, 116, 186-201.

Chiaradia, A.P.M., Gill, E., Montenbruck, O., Kuga, H.K. and Prado, A.F.B.A. (2000). Algorithms for OnBoard Orbit Determination using GPS OBODE-GPS, DLR - GSOC TN 00-04.

Czopek, F.M. and Shollenberger, S. (1993). Description and performance of the GPS Block I and II L-Band antenna and link budget. ION-GPS 93, The Institute of Navigation, Salt Lake City, pp. 37-43.

Engel, U. (2008). Improving Position Accuracy by Combined Processing of Galileo and GPS Satellite Signals. IEEE, 11th International Conference on Information Fusion, Cologne, Germany, pp. 1-8.

Erker, S., Tholert, S., Furthner, J. and Meurer, M. (2009). L5 - The New GPS Signal. IAIN 2009, Stockholm, Sweden.

ESA. (2010). Galileo SIS ICD Issue $1 \cdot 1$.

Groves, P.D. (2013). Principles of GNSS, Inertial, and Multisensor Integrated Navigation systems, Artech House.

Habib, T.M.A. (2013). Simultaneous spacecraft orbit estimation and control based on GPS measurements via extended Kalman filter. The Egyptian Journal of Remote Sensing and Space Sciences, 16, 11-16.

Kalman, R.E. (1960). A New Approach to Linear Filtering and Prediction Problems. ASME Transactions, Series D: Journal of Basic Engineering, 82, 35-45.

Kaplan, E.D. and Hegarty, C.J. (2006). Understanding GPS: Principles and Applications, Artech House.

Lai, K.L. and Crassidis, J.L. (2006). Generalizations of the Complex-Step Derivative Approximation. American Institute of Aeronautics and Astronautics.

Litva, J. (1996). Digital Beamforming in Wireless Communications, Artech House.

Martins, J.R.R.A., Sturdza, P. and Alonso, J.J. (2003). The Complex-Step Derivative Approximation. $A C M$ Transactions on Mathematical Software, 29(3), 245-262.

McDonald, K.D. and Hegarty, C. (2000). Post-Modernization GPS Performance Capabilities, Proceedings of ION 56th Annual Meeting, San Diego, California, USA (Institute of Navigation), pp. 242-249.

Miller, J. (2011). Enabling a Fully Interoperable GNSS Space Service Volume. 6th International Committee on GNSS (ICG), Tokyo, Japan.

Montenbruck, O. and Gill, E. (2000). Satellite Orbits: Models, Methods, Applications, Springer.

Palmerini, G.B., Sabatini, M. and Perrotta, G. (2009). En route to the Moon using GNSS signals. Acta Astronautica, 64(4), 467-483.

Pardal, P.C.P.M., Kuga, H.K. and Vilhena de Moraes, R. (2009). Non Linear Sigma Point Kalman Filter Applied to Orbit Determination Using GPS Measurements. Proceedings of the 22nd International Technical Meeting of The Satellite Division of the Institute of Navigation (ION GNSS 2009) Savannah, Georgia, USA, pp. 1478-1485.

Powell, T. D., Martzen, P.D., Sedlacek, S. B., Chao, C.-C., Silva, R., Brown, A. and Belle, G. (1999). GPS Signals in a Geosynchronous Transfer Orbit: "Falcon Gold" Data Processing, Proceedings of the 1999 National Technical Meeting of The Institute of Navigation, San Diego, CA, pp. 575-585.

Silva, P.F., Lopes, H.D., Peres, T.R., Silva, J.S., Ospina, J., Cichocki, F., Dovis, F., Musumeci, L., Serant, D., Calmettes, T., Pessina, I. and Perelló, J.V. (2013), Weak GNSS Signal Navigation to the Moon, Proceedings of the 26th International Technical Meeting of The Satellite Division of the Institute of Navigation (ION GNSS+2013), Nashville, TN, pp. 3357-3367.

Spirent. (2012). SimGen Software User Manual.

Unwin, M., Van Steenwijk, R. De Vos, Blunt, P., Hashida, Y., Kowaltschek, S. and Nowak, L. (2013). Navigating Above the GPS Constellation-Preliminary Results from the SGR-GEO on GIOVE-A, Proceedings of the 26th International Technical Meeting of The Satellite Division of the Institute of Navigation (ION GNSS+2013), Nashville, TN, pp. 3305-3315.

Van Diggelen, F. (2009). A-GPS: Assisted GPS, GNSS and SBAS, Artech house.

Woodward, M. and Folta, D.a.W.D. (2009). ARTEMIS: The First Mission to the Lunar Libration Orbits. Toulouse, France, 21st International Symposium on Space Flight Dynamics.

Wu, A. (2002). Predictions and Field Measurements of the GPS Block IIR L1 and L2 Ground Powers, Proceedings of the 2002 National Technical Meeting of The Institute of Navigation, San Diego, CA, pp. 931-938. 